\title{
Effect of COVID-19 Stay-at-Home Restrictions on Parent Reported Symptom Severity and Adaptive Functioning of Youth with ASD
}

\author{
Christopher Lopata ${ }^{1}$ (D) Jonathan D. Rodgers ${ }^{1}$. James P. Donnelly ${ }^{1}$. \\ Marcus L. Thomeer ${ }^{1}$. Jennifer Lodi-Smith ${ }^{1}$. Zoe L. Gionis ${ }^{1}$. \\ Samantha L. Andrews ${ }^{1}$. Christian J. Rajnisz ${ }^{1}$
}

Accepted: 9 July 2021 / Published online: 24 July 2021

(c) The Author(s), under exclusive licence to Springer Science+Business Media, LLC, part of Springer Nature 2021

\begin{abstract}
This study assessed the potential short-term effects of COVID-19 stay-at-home restrictions on ratings of ASD and comorbid symptoms severity and adaptive functioning of 69 youth, ages 8-16 years with ASD without intellectual disability. Parent/caregiver ratings were being collected in fall and spring over approximately two years when the restrictions were imposed four months prior to the final data collection point. Results indicated no significant changes in parent/caregiver ratings of ASD symptom severity, comorbid symptoms severity, social skills, or adaptive behaviors following the stay-at-home restrictions and little variability across the four data collection points. Although findings suggested minimal short-term effects on these symptoms and adaptive skills, ongoing monitoring is needed to assess longerterm impacts.
\end{abstract}

Keywords COVID-19 restrictions · ASD symptoms · Comorbid symptoms · Adaptive skills

Youth with autism spectrum disorder (ASD) are defined by social impairments and restricted and repetitive behaviors/interests that significantly interfere with daily functioning (American Psychiatric Association [APA], 2013). Their social impairments are complex (including deficits in basic skills and social-cognitive knowledge/understanding; Bellini et al., 2014), persist into adulthood, and contribute to poor long-term outcomes (limited employment, social relationships, and independence; Barnhill, 2007). Restricted and repetitive behaviors/interests also

Christopher Lopata

lopatac@canisius.edu

1 Institute for Autism Research, Canisius College, 2001 Main Street, Buffalo, NY 14208, USA 
interfere with daily functioning and can include a strong adherence to routines and need for sameness (e.g., cognitive rigidity, significant distress resulting from minor changes in routine or the environment), and sensory sensitivities (e.g., negative reactions and behaviors in response to certain sensory input such as particular sounds, noise level, lighting; APA, 2013). Many studies have also documented adaptive skills deficits of youth with ASD including among those without intellectual disability (ID; McDonald et al., 2016).

Youth with ASD also frequently exhibit a range of comorbid symptoms (APA, 2013). Common externalizing problems have included inattention, hyperactivity, oppositional behaviors, and conduct problems (Gadow et al., 2006; Lecavalier, 2006). Internalizing problems are also common including symptoms of anxiety, depression, worry, etc. (Sukhodolsky et al., 2008; White et al., 2009). These externalizing and internalizing symptoms have been found across the ASD population including among youth with ASD without ID (e.g., Nasca et al., 2020). Although comorbid symptoms/behaviors are common across the ASD population, some have found variability in their expression and/or type based on intellectual ability (IQ $\leq 70$ versus IQ $>70$ ), which may be due to factors such as selfawareness, social knowledge, communication ability, etc. (Sukhodolsky et al., 2008; White et al., 2009). Given the potential implications of IQ and language ability, there is a need to study comorbid symptoms in more homogeneous samples including youth with ASD without ID (Nasca et al., 2020).

The complex nature of the core ASD and comorbid symptoms requires effective interventions. Public schools constitute a primary source of services for youth with ASD (Iadarola et al., 2018), with many students with ASD without ID placed in mainstream and integrated classrooms (Mayes et al., 2011; McDonald et al., 2019). Although youth with ASD often have difficulty adjusting to new routines/schedules at the beginning of the school year, the adjustment can be facilitated with structured environments and routines (Mazefsky \& Herrington, 2014; Tsai, 2006). Once routines/schedules are established, disruptions can negatively affect core ASD symptoms, comorbid problems, and social and adaptive functioning (APA, 2013). The COVID-19 pandemic caused major disruptions to both school and home routines and environments owing to stay-at-home restrictions and school closings. These not only abruptly changed routines but children with ASD in states with such restrictions experienced disruptions in their school interventions and services.

This unprecedented pandemic provided a unique opportunity to study how ASD and comorbid symptoms severity and social and adaptive skills may have been impacted for youth with ASD without ID. This natural experiment was possible due to a 2-year study that was underway tracking the symptoms and functioning of a sample of youth with ASD without ID. Data were already collected for three time points (year one fall and spring and year two fall) when stay-at-home restrictions were imposed four months prior to the end of the school year (final data collection point). The restrictions were uniform statewide and included closing of schools and stay-at-home requirements for all children. This study assessed parent/caregiver ratings of symptom severity and social and adaptive skills following the stay-at-home restrictions. 


\section{Methods}

\section{Participants}

Sixty-nine youth, ages 8-16 years, with ASD without ID were rated by their parents/caregivers. The sample was recruited from a group of 90 youth and parents/ caregivers participating in a separate study tracking the symptoms and adaptive functioning of these youth over approximately two years. Eligibility criteria for that study were a Wechsler Intelligence Scale for Children- $4^{\text {th }}$ Edition (WISCIV) short-form IQ $>70$ (and VCI or PRI $\geq 80$; Wechsler, 2003), short-form Comprehensive Assessment of Spoken Language (CASL; Carrow-Woolfolk, 1999) expressive or receptive language score $\geq 75$, and diagnosis of ASD (confirmed via the Autism Diagnostic Interview-Revised [ADI-R]; Rutter et al., 2003). The participation rate $(77 \%)$ was considered high given increased demands on parents/ caregivers due to the stay-at-home restrictions; however, the group of respondents and non-respondents were compared on child and family characteristics to rule out systematic differences that might have affected results. No significant differences were found between respondents and non-respondents based on child age, IQ, language level, diagnostic ASD symptoms, or Time 1 outcome measures, or parent education $(p s>0.05)$. See Table 1 for demographic data.

\section{Measures}

Social Responsiveness Scale, $2^{\text {nd }}$ Ed., School Age Form (SRS-2) The SRS-2 (Constantino $\&$ Gruber, 2012) is a 65-item objective measure of ASD-related symptoms including social-communication deficits and circumscribed and repetitive behaviors/interests. Parents rate the frequency of behaviors on a scale of 1 (not true) to 4 (almost always true), with higher scores indicating greater symptoms/severity. The total score has internal consistency of 0.94-0.97. Moderate-to-high correlations are reported with other ASD diagnostic measures and the test discriminates between ASD and nonASD samples. The SRS-2 also yields two index scores (Social Communication and Interaction [SCI] and Restricted Interests and Repetitive Behavior [RRB]) that reflect the two major symptom domains of ASD.

Behavior Assessment System for Children, Third Edition-Parent Rating Scales (BASC-3-PRS) The BASC-3-PRS (Reynolds \& Kamphaus, 2015) assesses behaviors across a variety of domains. Parents rate items from 0 (never) to 3 (almost always). This study used the Externalizing (EXT), Internalizing (INT), and Adaptive Skills (AS) composites to assess comorbid symptom severity and adaptive functioning. On the EXT and INT composites, higher scores indicate higher comorbid symptom levels, and on the AS composite, lower scores indicate lower adaptive functioning. Internal consistency reliabilities for the EXT composite ranged from 0.93-0.96, INT ranged from 0.93-0.96, and AS ranged from 0.96-0.97. Moderate correlations are 
Table 1 Descriptive statistics for demographic and sample characteristics

\begin{tabular}{|c|c|c|}
\hline Characteristic & Participants $(n=69)$ & Non-respondents $(n=21)$ \\
\hline & $M(S D)$ & $M(S D)$ \\
\hline Parent education & $15.91(2.11)$ & $15.36(2.47)$ \\
\hline \multicolumn{3}{|l|}{ Child } \\
\hline Age (yrs.) & $12.32(1.56)$ & $12.32(1.98)$ \\
\hline WISC-IV IQ (short-form) & $102.52(14.83)$ & $102.20(12.54)$ \\
\hline WISC-IV VCI (short-form) & $100.82(14.67)$ & $103.32(15.99)$ \\
\hline WISC-IV PRI (short-form) & $103.79(18.08)$ & $100.54(10.73)$ \\
\hline CASL expressive language (short-form) & $95.85(14.95)$ & $96.15(14.60)$ \\
\hline CASL receptive language (short-form) & $100.83(17.16)$ & $102.10(18.59)$ \\
\hline ADI-R social interactions & $19.07(6.03)$ & $17.38(4.35)$ \\
\hline ADI-R communication & $14.84(4.66)$ & $14.86(4.54)$ \\
\hline \multirow[t]{2}{*}{ ADI- $R$ repetitive Behavior } & $6.00(1.92)$ & $6.43(2.18)$ \\
\hline & $n$ (within group \%) & $n$ (within group \%) \\
\hline Sex (male) & $62(90 \%)$ & $20(95 \%)$ \\
\hline Race/ethnicity (Caucasian) & $66(96 \%)$ & $20(95 \%)$ \\
\hline \multicolumn{3}{|l|}{ Parental job disruption } \\
\hline No change & $54(78 \%)$ & unknown \\
\hline Reduced hours & $3(4 \%)$ & unknown \\
\hline Job loss & $12(17 \%)$ & unknown \\
\hline \multicolumn{3}{|l|}{ Child psychosocial support services ${ }^{\mathrm{a}}$} \\
\hline None & $47(68 \%)$ & unknown \\
\hline Counseling (phone/video) & $18(26 \%)$ & unknown \\
\hline Social skills group (video) & $4(6 \%)$ & unknown \\
\hline
\end{tabular}

$N=69$. WISC-IV, Wechsler Intelligence Scale for Children-4th Edition; VCI, Verbal Comprehension Index; PRI, Perceptual Reasoning Index; CASL, Comprehensive Assessment of Spoken Language; ADI$R$, Autism Diagnostic Interview-Revised

${ }^{a}$ Indicates psychosocial support services received during the stay-at-home period

reported between these scales and comparable scales on other well-known rating scales.

Adapted Skillstreaming Checklist (ASC) The ASC (Lopata et al., 2008) measures social skills and behaviors of youth with ASD without ID. Across the 38 items, 32 assess social/social-communication skills and 6 assess behavioral regulation and flexibility. Parents rate each item from 1 (almost never) to 5 (almost always), with higher total scores indicating greater use of the prosocial skills. The ASC total score, based on parent ratings of youth with ASD without ID (Lopata et al., 2017) yielded an internal consistency of 0.92 . Validity was supported in significant inverse correlations with ratings of ASD symptoms and problem behaviors and positive correlations with prosocial/adaptive skills on established scales. 


\section{Procedures}

This study was approved by the Institutional Review Board and written parental consent and child assent were obtained. Parent/caregiver ratings were being collected in fall and spring over approximately two years when the stay-at-home restrictions were imposed four months before the final data collection point. The study also included teacher ratings and testing of the children's social-cognitive skills; however, data for the final collection point could not be collected from teachers (due to schools being closed) or children (due to restrictions on direct contact with the children). Parents/caregivers were invited to complete the rating forms describing the children's functioning four months after the stay-at-home restrictions were instituted. As such, parent/caregiver ratings reflected child functioning primarily in the home setting. Two items were added to the assessment: one asked about parental job disruption; and the other asked whether the child received psychosocial support services during the shutdown. Collecting ratings twice per year over approximately two years (versus one year at fall and spring) also allowed for ruling-out of seasonal variation that might have influenced results.

Children were rated by the same primary parent/caregiver at each time point. Each rating form was checked upon return for completeness and any errors (multiple endorsements, omitted items, etc.) were immediately corrected by checking with the informant. Each protocol was scored independently by two research assistants, with any scoring discrepancies resolved by a third scorer. All study data were entered into a database and independently checked by a second member of the team, with discrepancies resolved by a third team member.

\section{Data Quality and Analysis Overview}

Analysis included assessment of data quality and missingness prior to examination of changes associated with the stay-at-home restrictions. Three analyses of the original sample of 90 families were conducted to determine the potential for bias if the 69 cases were to be used. Next, the analysis tested the potential impact of the stayat-home restrictions on Time 4 ratings. Specifically, the possibility of change in the pattern of scores was tested as the difference between the post stay-at-home restrictions (Time 4) and the prior three assessments. This involved plots of the seven outcomes over time, repeated measures analysis of variance for the overall time effect, and paired $t$-tests of the Time 4 assessment with the mean of the prior three. The final analysis examined whether parental employment disruptions or child psychosocial support services might be associated with scores at Time 4. Effect sizes with $95 \%$ confidence intervals were calculated. As an exploratory study, the alpha error rate was 0.05 for all analyses.

Distributions of the seven outcome variables were examined for outliers, skewness and kurtosis. There were no deviations from normality and no values that exceeded 3 SD on the SRS-2 Total, SRS-2 SCI, SRS-2 RRB, BASC-3 AS, or ASC. Three participants had scores slightly above $3 S D$ on the BASC-3 EXT 
scale, and a different participant had two high scores on the BASC-3 INT scale. These scores were considered valid and retained in the analysis. There were no cases of outlying scores only at the Time 4 assessment (which may have indicated post stay-at-home restrictions impact).

As noted, missing data in the sample of 90 was assessed in several ways. First, missing data was examined at each time point. At Time 1, data from one family was missing (1\%) and there was no missing data at Times 2 or 3 . At Time 4 (post stay-at-home restrictions), data were missing from 21 families (23\%; did not participate). A series of comparative analyses was conducted to examine whether missingness at Time 4 was associated with child or family variables. Missingness at Time 4 was compared to child sex, ethnicity, and scores on the screening tests (WISC-IV, CASL, and ADI-R) and outcomes (SRS-2 Total and subscales, BASC-3 composites, and ASC). Parent education was also compared. These univariate chi-square and $t$-tests showed no evidence of an association between missing at Time 4 and any of the sample characteristics. Biserial correlations between each outcome variable at Time 1 with missingness at Time 4 were calculated. The largest correlation was 0.06 and the average among the seven correlations was 0.003 . Next, logistic regression analysis was used to determine whether the set of sample characteristics might predict missingness at Time 4 . This analysis indicated that the set of 12 sample characteristics did not significantly predict missingness at Time $4\left(\chi^{2}=8.46, d f=11, p=0.67\right)$. These analyses were interpreted to mean that the impact of the stay-at-home restrictions could be studied with the sample of 69 without risk of sampling bias due to non-participation.

\section{Results}

Table 1 shows demographic and sample characteristics as well as two variables included as stay-at-home restrictions impacts (i.e., parental employment disruption and child psychosocial support services). Table 2 presents descriptive statistics for the outcome variables, with omnibus $F$-tests for time, paired $t$-tests and associated $p$-values and effect sizes. All tests were non-significant and the planned contrasts yielded negligible effect sizes ( $d$ s 0.01-0.12) indicting no evidence of COVID-19 stay-at-home restrictions impact on any outcome variable. The data was recast slightly in Fig. 1 to show the pattern of symptoms and skills across the four assessments; it includes data on ASD symptom severity (SRS-2 Total), comorbid symptom severity (BASC-3 EXT and INT composites), social/ social-communication skills (ASC), and broader adaptive behaviors (BASC-3 AS composite). The pattern depicts relatively little variability across the four time points, and shows no evidence of post stay-at-home restrictions effects. The final analysis assessed whether parental job disruption or child psychosocial support services during the shutdown were associated with variability in Time 4 ratings. Independent samples $t$-tests showed no difference on any of the seven outcomes for either job disruption or child psychosocial support ( $p s>0.05)$. 


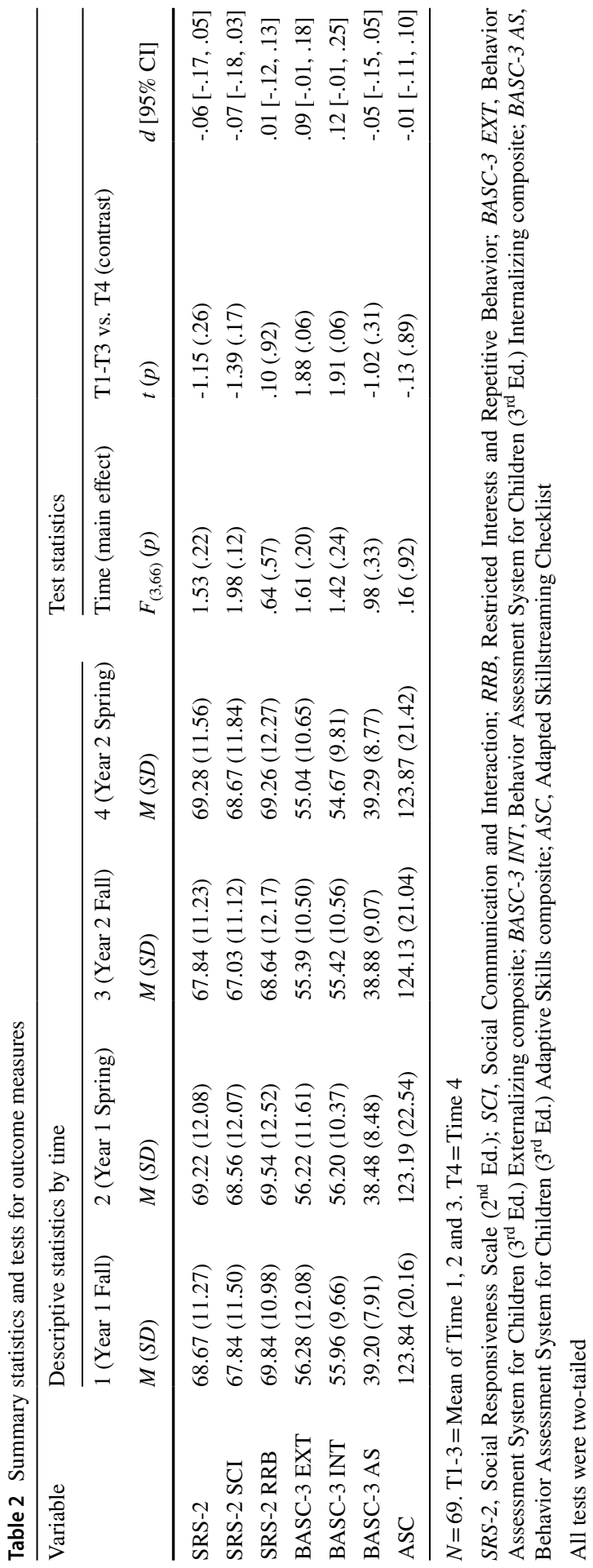




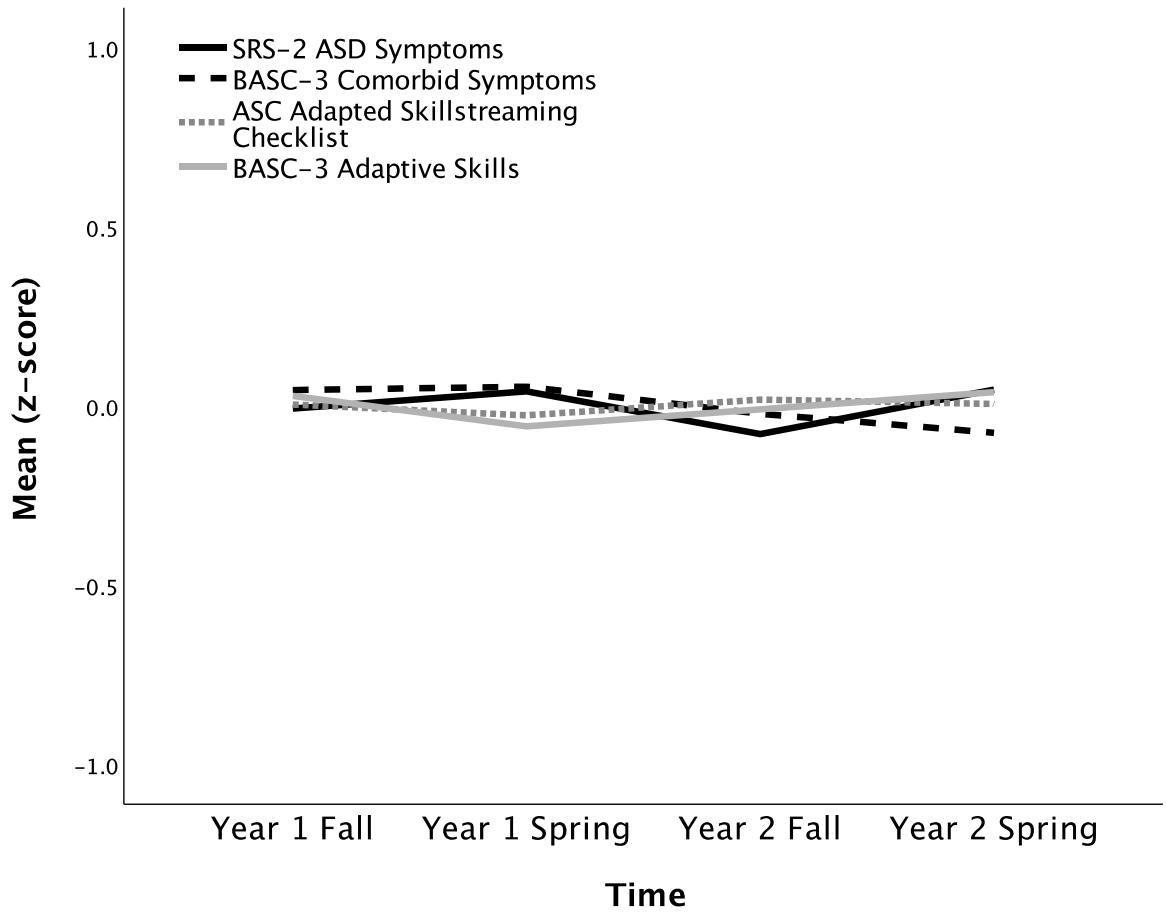

Fig. 1 Plot of ASD symptoms, comorbid symptoms, and adaptive skills by time. Note. SRS-2=Social Responsiveness Scale ( $2^{\text {nd }}$ Ed.); BASC-3 Comorbid Symptoms = Behavior Assessment System for Children $\left(3^{\text {rd }}\right.$ Ed.) Externalizing and Internalizing z-score mean

\section{Discussion}

Youth with ASD demonstrate a strong preference for predictability and routines and their symptoms and skills can be affected by environmental circumstances/conditions. Specifically, changes and disruptions to established routines and expectations may lead to an exacerbation of ASD and comorbid symptoms severity and/or a decline in prosocial skills and adaptive behaviors. This study assessed the short-term effects of COVID-19 stay-at-home restrictions on the symptoms and functioning of youth with ASD without ID as rated by parents/ caregivers. Parent/caregiver ratings were being collected during an approximate 2-year period when stay-at-home restrictions were imposed four months prior to the final data collection point; this study compared the final data collection point (post stay-at-home restrictions) to the three prior data collection points (pre-restrictions).

Statewide COVID-19 stay-at-home restrictions abruptly changed the school and home routines of youth in the sample. Although all the children reportedly received academic instruction in some form (e.g., handouts/worksheet packets, online, virtual), most did not receive psychosocial support services during the stay-at-home period. Additionally, $>20 \%$ of parents/caregivers experienced job 
disruption (reduced hours or job loss). Although the stress associated with such changes might be expected to have a negative effect on the functioning of youth with ASD without ID, results suggested little impact. Based on parent/caregiver ratings, youth in this sample demonstrated no increase in overall ASD symptom severity (or specific symptom severity involving social communication or restricted and repetitive behaviors) or in externalizing or internalizing symptoms severity following the shutdown, and limited variability across the time points. This might be considered surprising as disruptions to routines and schedules have been associated with increased ASD and comorbid symptoms (APA, 2013).

Additionally, there was no significant change in parent/caregiver ratings of social skills or broader adaptive behaviors. This finding was interesting given that youth in the sample did not have typical social contact with their school-based peers and only a small minority received psychosocial support services during the stay-athome restrictions. These youth would have had fewer social opportunities following the shutdown than would have been afforded in the school environment. The lack of decline is even more interesting given the stability of parent/caregiver ratings of their social skills across the four time points. Broader adaptive functioning also appeared to be unaffected and was similarly consistent across the approximate 2-year time period.

The overall pattern of parent/caregiver ratings suggests that youth with ASD without ID in the sample were largely unaffected over this time period (shortterm) by the COVID-19 stay-at-home restrictions and disruptions to routines. Although the reason(s) for lack of negative impacts is unknown, there are several possibilities. One possible explanation is that the negative effect(s) of disruptions to routines was not as distressing as anticipated for these youth. A second possibility is that changes in symptoms or adaptive skills may take longer than four months to materialize and/or for parents/caregivers to fully recognize. A third possible, and more likely, explanation involves the school environment itself, which poses many challenges for youth with ASD including those without ID. For example, school environments present increased social demands and youth with ASD often struggle to interpret social cues and effectively engage with peers (Bauminger-Zviely, 2014). These demands can be even greater for youth with ASD without ID who receive much or all of their education in mainstream and integrated classrooms (Mayes et al., 2011; McDonald et al., 2019). Poor social competence also makes these youth vulnerable to bullying, which is commonly reported by these individuals (Bauminger-Zviely, 2014). Further, school environments are characterized by unpredictable events (e.g., changes in schedules, exposure to unfamiliar peers and staff, etc.; Ashburner et al., 2010) which can run counter to the predictability preferred by these youth. School environments also present a range of sensory stimuli that youth with ASD may find distressing and are unavoidable (e.g., crowded hallways/cafeterias, loud noises, bright lighting; Mazefsky \& Herrington, 2014). Although the COVID-19 stay-at-home restrictions abruptly changed the established routines of these youth more than halfway through the school year, it is possible that this disruption was no more distressing than the challenges encountered on a regular basis in the school setting. The reduced social demands and familiarity of the home environment (compared to 
schools) may have countered the disruptions imposed by the COVID-19 school closings. It is important to recognize that the current findings are based on parent/ caregiver ratings of the children's symptoms/functioning primarily in the home setting as statewide stay-at-home restrictions prevented community- and schoolbased activities.

Despite the lack of short-term adverse impacts, ongoing disruptions to schooling, lack of social opportunities, and/or stay-at-home restrictions may have negative effects not captured four months after this shutdown. As such, it is necessary to monitor the longer-term effects and how those might be related to ongoing stay-at-home restrictions or the various formats that will be used to deliver educational services for these youth (online, hybrid online and in-person classes, or all in-person). This will be critical as schools are a primary source of services for many youth with ASD (Iadarola et al., 2018) and lack of access to effective psychosocial and educational programming may have longer-term consequences.

This study provides insights into the short-term effects of COVID-19 stay-athome restrictions and it had a number of strengths (e.g., relatively large, functionally-homogeneous, and well-characterized sample, testing of effects from a naturally occurring event that could not be experimentally manipulated, testing across approximately two years, etc.), however several limitations warrant mention. One limitation involved the sample that was largely male and Caucasian, and only included youth with ASD without ID. As such, the results cannot necessarily be generalized to those outside these parameters. A second limitation involved the use of parent/caregiver ratings only (teacher ratings could not be collected and testing of the youth could not be done due to shutdown restrictions). As a result, this study does not provide insight into potential changes in symptom severity or adaptive skills in the school environment, and/or changes in social-cognitive skills of those in the sample. Additionally, this study only tested the short-term (four months) impacts. Overall, the findings provide important information but need to be interpreted within the context of the raters (parents/caregivers), setting (home), measure type (rating scales), and time period (four months) of this study. Discrepancies can be observed between informants and/or measure types within the same or across settings, as well as over time and the current results may not generalize to other informants, settings, measurement methods, or time frames. Lastly, no adjustment was applied for the multiple comparisons; however, the statistical conclusions would have been the same regardless of whether the correction was applied.

Given these limitations, investigators should continue to monitor the impacts of these restrictions in longitudinal studies that also include teacher ratings and testing of the youth. It will also be important to monitor the manner in which schools reopen to determine if a particular format (online, hybrid, in-person) is more effective for youth with ASD. In addition, it may be useful to interview youth in the sample, parents/caregivers, and teachers to gain insight into how they perceive the impacts (benefits and consequences) of stay-at-home restrictions and school closings. Lastly, studies of parent/caregiver experiences, stress, and functioning related to COVID-19 restrictions for the children would provide valuable insights into broader impacts. 
Funding The research reported in this article was supported by Department of Education, Institute of Education Sciences Grant R324A180005. Findings and conclusions are those of the authors and do not necessarily reflect the views of the funding agency.

\section{Declarations}

Conflict of Interest The authors declare that they have no conflict of interest.

Ethics Approval This study was approved by the Institutional Review Board at Canisius College and was performed per the approved protocol.

Informed Consent Written informed parental consent and child assent were obtained for participation in the study.

\section{References}

American Psychiatric Association. (2013). Diagnostic and statistical manual of mental disorders (5th ed.). American Psychiatric Association.

Ashburner, J., Ziviani, J., \& Rodger, S. (2010). Surviving in the mainstream: Capacity of children with autism spectrum disorders to perform academically and regulate their emotions and behavior at school. Research in Autism Spectrum Disorders, 4, 18-27. https://doi.org/10.1016/j.rasd. 2009.07.002

Barnhill, G. P. (2007). Outcomes in adults with Asperger syndrome. Focus on Autism and Other Developmental Disabilities, 22, 116-126. https://doi.org/10.1177/10883576070220020301

Bauminger-Zviely, N. (2014). School-age children with ASD. In F. R. Volkmar, S. J. Rogers, R. Paul, \& K. A. Pelphrey (Eds.), Handbook of autism and pervasive developmental disorders: Vol. 1. Diagnosis, development, and brain mechanisms (4 ${ }^{\text {th }}$ ed., pp. 148-175). John Wiley \& Sons.

Bellini, S., Gardner, L., \& Markoff, K. (2014). Social skill interventions. In F. R. Volkmar, S. J. Rogers, R. Paul, \& K. A. Pelphrey (Eds.), Handbook of autism and pervasive developmental disorders: Vol. 2. Assessment, interventions, and policy ( $4^{\text {th }}$ ed., pp. 887-906). John Wiley \& Sons.

Carrow-Woolfolk, E. (1999). Comprehensive Assessment of Spoken Language. American Guidance Service.

Constantino, J. N., \& Gruber, C. P. (2012). Social Responsiveness Scale, Second Edition (SRS-2). Western Psychological Services.

Gadow, K. D., DeVincent, C. J., \& Pomeroy, J. (2006). ADHD symptom subtypes in children with pervasive developmental disorder. Journal of Autism and Developmental Disorders, 36, 271283. https://doi.org/10.1007/s10803-005-0060-3

Iadarola, S., Shih, W., Dean, M., Blanch, E., Harwood, R., Hetherington, S., ... \& Smith, T. (2018). Implementing a manualized, classroom transition intervention for students with ASD in underresourced schools. Behavior Modification, 42, 126-147.

Lecavalier, L. (2006). Behavioral and emotional problems in young people with pervasive developmental disorders: Relative prevalence, effect of subject characteristics, and empirical classification. Journal of Autism and Developmental Disorders, 36, 1101-1114. https://doi.org/10.1007/ s10803-006-0147-5

Lopata, C., Rodgers, J. D., Donnelly, J. P., Thomeer, M. L., McDonald, C. A., \& Volker, M. A. (2017). Psychometric properties of the Adapted Skillstreaming Checklist for high-functioning children with ASD. Journal of Autism and Developmental Disorders, 47, 2723-2732. https://doi.org/10. 1007/s10803-017-3189-y

Lopata, C., Thomeer, M. L., Volker, M. A., Nida, R. E., \& Lee, G. K. (2008). Effectiveness of a manualized summer social treatment program for high-functioning children with autism spectrum disorders. Journal of Autism and Developmental Disorders, 38, 890-904. https://doi.org/ 10.1007/s 10803-007-0460-7 
Mayes, S. D., Calhoun, S. L., Murray, M. J., \& Zahid, J. (2011). Variables associated with anxiety and depression in children with autism. Journal of Developmental and Physical Disabilities, 23, 325-337. https://doi.org/10.1007/s10882-011-9231-7

Mazefsky, C. A., \& Herrington, J. D. (2014). Autism and anxiety: Etiologic factors and transdiagnostic processes. In T. E. Davis, S. W. White, \& T. H. Ollendick (Eds.), Handbook of autism and anxiety (pp. 91-103). Springer.

McDonald, C. A., Donnelly, J. P., Alguire-Feldman, A. L., Rodgers, J. D., Lopata, C., \& Thomeer, M. L. (2019). Special education service use by children with autism spectrum disorder. Journal of Autism and Developmental Disorders, 49, 2437-2446. https://doi.org/10.1007/s10803-019-03997-z

McDonald, C. A., Lopata, C., Nasca, B., Donnelly, J. P., Thomeer, M. L., \& Rodgers, J. D. (2016). ABASII adaptive profiles and correlates in samples of children with HFASD and LFASD. Journal of Developmental and Physical Disabilities, 28, 769-783. https://doi.org/10.1007/s10882-016-9508-y

Nasca, B. C., Lopata, C., Donnelly, J. P., Rodgers, J. D., \& Thomeer, M. L. (2020). Sex differences in externalizing and internalizing symptoms of children with ASD. Journal of Autism and Developmental Disorders, 50, 3245-3252. https://doi.org/10.1007/s10803-019-04132-8

Reynolds, C. R., \& Kamphaus, R. W. (2015). Behavior Assessment System for Children (3rd ed.). Pearson.

Rutter, M., LeCouteur, A., \& Lord, C. (2003). Autism Diagnostic Interview-Revised. Western Psychological Services.

Sukhodolsky, D. G., Scahill, L., Gadow, K. D., Arnold, E., Aman, M. G., McDougle, C. J., ... \& Vitiello, B. (2008). Parent-rated anxiety symptoms in children with pervasive developmental disorders: Frequency and association with core autism symptoms and cognitive functioning. Journal of Abnormal Child Psychology, 36, 117-128. https://doi.org/10.1007/s10802-007-9165-9

Tsai, L. Y. (2006). Diagnosis and treatment of anxiety disorders in individuals with autism spectrum disorder. In M. G. Baron, J. Groden, G. Groden, \& L. P. Lipsitt (Eds.), Stress and coping in autism (pp. 388-440). Oxford University Press.

Wechsler, D. (2003). Wechsler Intelligence Scale for Children (4th ed.). The Psychological Corporation.

White, S. W., Oswald, D., Ollendick, T., \& Scahill, L. (2009). Anxiety in children and adolescents with autism spectrum disorders. Clinical Psychology Review, 29, 216-229. https://doi.org/10.1016/j.cpr. 2009.01.003

Publisher's Note Springer Nature remains neutral with regard to jurisdictional claims in published maps and institutional affiliations. 